\title{
Features of the formation of urban heat islands effects in tropical climates and their impact on the ecology of the city
}

\author{
Minh Tuan Le ${ }^{1}$, Nguyen Anh Quan Tran ${ }^{2, *}$ \\ ${ }^{1}$ Moscow State University of Civil Engineering, Yaroslavskoye Shosse, 26, Moscow, 129337, Russia \\ ${ }^{2}$ Peter the Great Saint-Petersburg Polytechnic University, Polytechnicheskaya, 29, Saint Petersburg, \\ 195251, Russia
}

\begin{abstract}
The cumulative heating in some urban areas due to the urban growth and its types of industry, energy and transport, is the effect of urban heat island (UHI). It is recognized as one of the characteristics of the urban climate. The temperature increase caused by the effect (UHI) affects the energy flow in urban ecological systems, creates an unusual urban climate. By studying the effects of climate factors, local building materials to optimize energy efficiency, urban landscape, UHI phenomenon could be significantly moderated.
\end{abstract}

\section{Introduction}

In early 19th century, scholar Luke Howard firstly measured and discussed UHI effect when studying urban climate in London, England [1]. The "heat islands" is stable over time in the form of a warm air dome with a higher temperature, elevated above cities with an area of $100 \mathrm{~km}^{2}$ or more. The average air temperature of the "heat island" in a large city or town is usually higher than its surrounding by $4{ }^{\circ} \mathrm{C}$, and by $8{ }^{\circ} \mathrm{C}$ or more at night.

A characteristic attribute of modern megacities, showed as a region of high air temperature in some parts of the city, is the urban heat island. The effect of a thermal island is understood as follows:

- The island of heat can be observed that temperatures remain elevated over certain areas of urban development (the physical mechanisms leading to its formation have been mainly studied in the mid-1980s).

- Most of the city represents a "plateau" of warm air with an increase in temperature towards the city center. The thermal homogeneousness of the "plateau" is disturbed by "discontinuities" of the surface between cool regions (parks, ponds) and heat generated regions such as industrial enterprises, energy calculations, transport system, density of building blocks. On the border of city, residential areas, industrial areas, conceivably there is a significant vertical temperature drop, sometimes reaching several degrees, called the "island of heat".

Urbanization is developing in tropical countries rapidly, urban design issues relating to climate change are necessary in this stage. Hanoi city is one of the fast-growing cities in north

${ }^{*}$ Corresponding author: Architect290587@gmail.com 
part of Vietnam. Unplanned and rapid urban development in Hanoi can change the microclimate in this city, cause changes in temperature and wind pattern. Nevertheless, rivers, lakes and wind speed can be lessened the intensity of heat island effect in urban areas. The modern materials can help to reduce the heat absorption in buildings, roads and mitigating the heat island effect. Therefore, this paper aims to show the existence of heat island effect in Hanoi city, its causes, impact from wind rule and modern materials, to reduce the urban heat island effect.

\section{Meteorology condition analysis of Hanoi city, Vietnam}

The Hanoi Meteorological Station has 2 observation posts:

Station №1 - "Lang” is located in the Dong Da area, at $105.51^{\circ}$ east longitude, $21.02^{\circ}$ north latitude, at an elevation about $5.97 \mathrm{~m}$ above sea level.

Station №2 - "Son Tay" is located in the Son Tay area at $105.30^{\circ}$ E, $21.08^{\circ} \mathrm{N}$, at an altitude of $16.40 \mathrm{~m}$ above the sea level.

According to the materials published by the meteorological station, Hanoi is located in the subarea AIII:

- $\quad$ Spring lasts from February to April (air temperature $18-22^{\circ} \mathrm{C}$ ).

- Summer (hot and rainy) lasts from May to August. The average air temperature is + $35^{\circ} \mathrm{C}$.

Autumn lasts from August to October. The average air temperature is $+22^{\circ} \mathrm{C}$.

- Winter lasts from November to January. In winter, cold winds blow from the northeast and are accompanied by drizzly rains and fogs. Air humidity often reaches $85-90 \%$, the average air temperature is not changing, around $+5^{\circ} \mathrm{C}$.

The city of Hanoi is located at $105.51^{\circ}$ east longitude, $21.02^{\circ}$ north latitude. The height

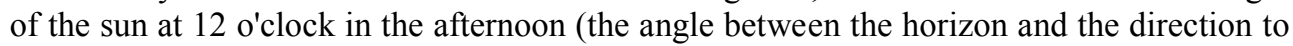
the sun) is $45.5^{\circ}$ in winter (22 December), and $92.5^{\circ}$ in the summer (22 June) (Fig. 1).

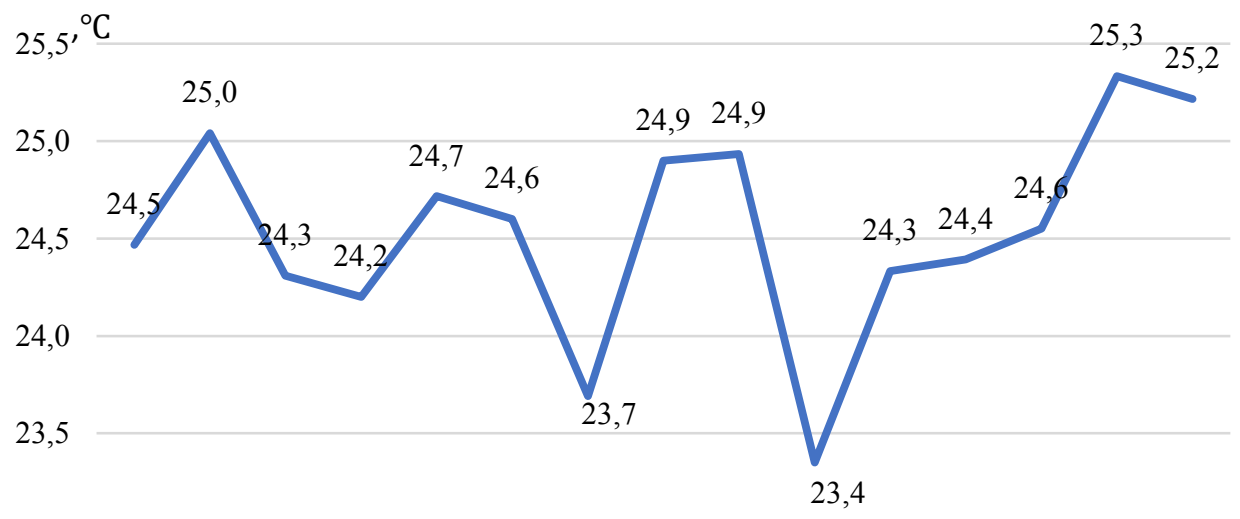

23,0

22,5

22,0

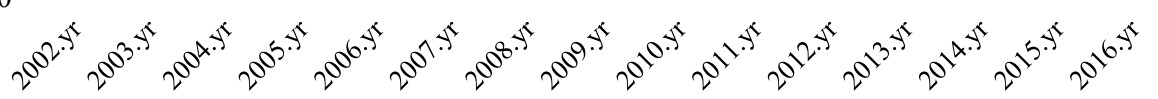

Fig. 1. Average temperature of Hanoi city from 2002 to 2016 (Station "Lang”, Hanoi city) [2]. 
The microclimate of the city is determined by the following main indicators: air temperature, solar radiation, precipitation, wind speed and direction.

In the summer, the sun's rays arrive in a steep angel at noon causing intensity of solar radiation reaches $908 \mathrm{~W}$. In winter, the maximum intensity of solar radiation is $804 \mathrm{~W}$. In both summer and winter, the scattered radiation is $45-50 \%$ of the total solar radiation. The average monthly number of hours of sun is shown in Fig. 2.

Furthermore, the microclimate is affected by: small-scale irregularities in the terrain, the character of the buildings (number of floors, occupants, urban plan layout), transport, the availability of industrial enterprises, landscaping and water areas. As the result of interaction, differences in microclimatic appear, specifically in temperature, humidity on the air surface layer, evaporation, wind direction and velocity, as well as "heat islands". These differences are greatest in quiet and sunny weather, but it will be least in the temperature with strong wind (buildings and other structures impact in the airflow strongly). Significant disturbances in temperature is also made by the layout of urban areas (direction of streets, location and size of areas, river flows, etc.).

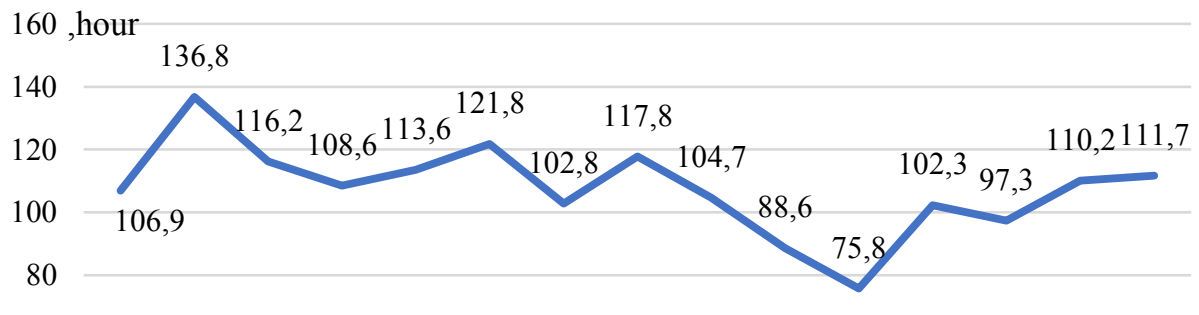

60

40

20

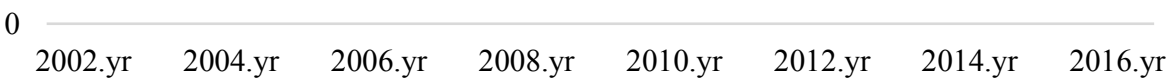

Fig. 2. Average sun hours from 2002 to 2016 (Station “Lang”, Hanoi city) [2].

In atmospheric phenomenon, the impact of wind directly affects human, through the combination by: wind with rain, wind with dust, strong wind (with speed more than $25 \mathrm{~m} / \mathrm{s}$ ). Beside the direct impact on human, it is necessary to consider the load of the wind on the buildings, the air pollution from traffic delivered by the wind and the environment.

\section{Method are based on the Landsat 8 satellite's images.}

Remote sensing, known as thermal sensing, involves electromagnetic waves with a wavelength of $3.5 \mu \mathrm{m}$ to $20 \mu \mathrm{m}$. Most remote sensing applications use a wavelength range of $8 \mu \mathrm{m}$ to $13 \mu \mathrm{m}$. The surface temperature is calculated from the thermal channel 10 Landsat 8 with a wavelength of $10.6 \mu \mathrm{m}-11.19 \mu \mathrm{m}$, a resolution of $100 \mathrm{~m}$.

The methodology is based on converting the temperature values from the gray value on the thermal channel 10 of the Landsat 8 satellite image. The implementation is performed by converting the numerical value into a spectral value $(\lambda)$, then convert this radiation value to a temperature value [3]. 


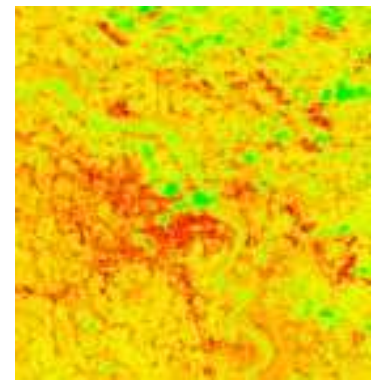

Fig. 3. Central city of Hanoi In the summer of 07/08/2017.

Int $42,08^{\circ} \mathrm{C}$

Lakes and rivers in Hanoi play an important role in the landscape of the city which help moderating the drain and reduce the temperature of the city in the summer. The space around the rivers and lakes is a place for cultural events. There are 111 lakes in the region of Hanoi city with a total area of 2186 hectares. In the city central region there are 24 lakes (765 hectares), which cover $10 \%$ of the urban area.

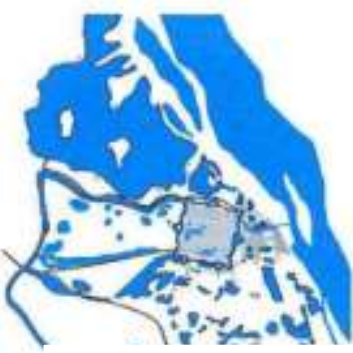

Feudal period

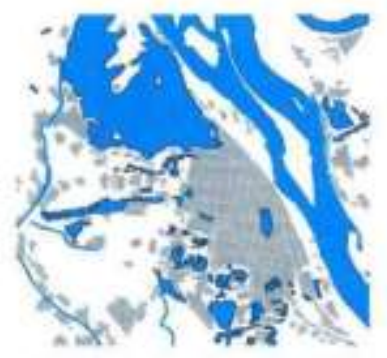

The period from 1954 to 1986

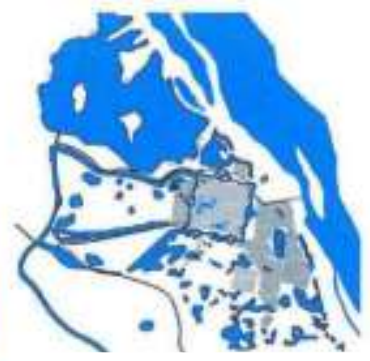

Colonial French Period

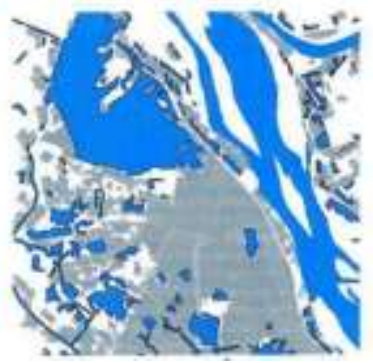

The period from 1986 to 1992

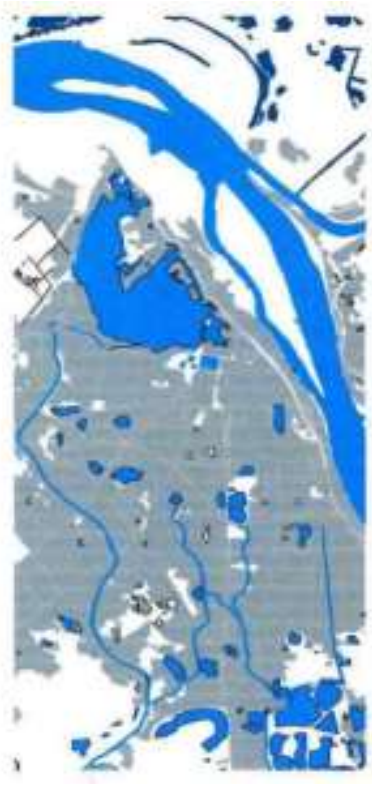

Present

Fig. 5. The system of rivers and lakes of Hanoi city by periods.

Rapid urbanization causes a decreasing in the area of lakes (Fig. 5). During spilling, rivers and lakes cause pollution in the environment around directly. The internal river system of the city becomes shallow as a result of urbanization. Water from central city's rivers is no longer used as a pure water for drinking because of heavy pollution. It is not only affecting people's health, but also changes the surrounding landscape. During the rainy season (July-August), some areas of Hanoi are often flooded. The flood in the city affects the environment, causing epidemics. 
In general, rivers and lakes could improve heat transporting process and bring fresh air to the city, so as to balance urban temperature. In addition, rivers and lakes could also separate urban thermal fields, reducing thermal radiation, alleviating thermal circulation and eliminating UHI effect. In summer, urban rivers and lakes are the main sources to reduce urban temperature in Hanoi city. Therefore, building planning and density along rivers and lakes will directly influence the effect of wind towards urban central areas. Areas adjacent to water zone with convenient traffic is beneficial to commercial activities and climatic improvement.

\section{Impact of natural wind with UHI effect}

The acknowledgement in climate studies showed that mitigating the effect of urban heat islands should notice on the sky view design factor, which plays an important role in urban geometry design. An investigation about the relationship between thermal performance and urban morphology with climatic responses [4], determined that looking a city structure can forecast the wind circulation which temperature variations show itself in turn. City morphology directly affects the movement of the wind within it, with the combination from design, shape, and infrastructures within it, as a result from studies. Claimed by another investigation, the connection between urban heat island intensity, wind speed and monitoring stations covered by cloud in Melbourne, Australia [5]. The primary results indicated that urban heat island values increased in calm winds and clear skies weather. An increase of wind speed by $1 \mathrm{~m} / \mathrm{s}$ in the summer can reduce $0.14^{\circ} \mathrm{C}$ in the intensity of heat island and by about $0.12^{\circ} \mathrm{C}$ when increasing the cloud cover on sky by an okta. Wind speed factor plays an important role in urban areas influencing the health, air quality, human's comfort and the energy consumption of the buildings [6,7]. In general, human feels comfortable with cool winds which can reduce the adverse effects of heat island in the microclimate. In Singapore, a wind velocity of $1-1.5 \mathrm{~m} / \mathrm{s}$ creates cooling effect which is equivalent to a $2{ }^{\circ} \mathrm{C}$ drop in temperature, as well as in the neighbor countries [8]. Additionally, the appropriate airflow can dissipate air pollution in [9]. Changing of wind pattern occurs as it flows through the urban regions [10]. Depending on the intensity of the built environment, winds are classified into three groups: isolated roughness flow, wake interference flow and skimming flow which are classified by the ratio $(\mathrm{H} / \mathrm{W})$ of building height $(\mathrm{H})$ to the distance between building arrays (W) [11]. Either weather condition or the occurrence of different flow regimes, including the wind speed and geometrical variables related to the buildings situated in urban settlements and open spaces are the causes of wind pattern [12]. Built forms determined the dimension, shape and placement of open spaces [13] and with wind induced airflow, it is significant to understand their relationship, especially in tropics where cooling effect of urban winds is beneficial.

Mostly, five important components that affect the condition of outdoor spaces are wind, solar radiation, air temperature, humidity and terrestrial radiation, help creating comfortable thermal surroundings [14]. These components can be rearranged and modified by elements in landscape. According to a research, there are total of 4 main ways to modify the microclimatic environment through landscape elements, especially green structures, including wind modification, relative humidity, incoming solar radiation and terrestrial radiation from the ground and other surfaces [14].

Human experience solar energy naturally in the form of visible light and infrared solar. About half of this energy is visible light and the other half is infrared [14]. Despite that, when solar energy arrives the earth, it is absorbed, transmitted or reflected by the ground. Energy nature sources are affected by the reflected radiation, as well as the thermal comfort. 


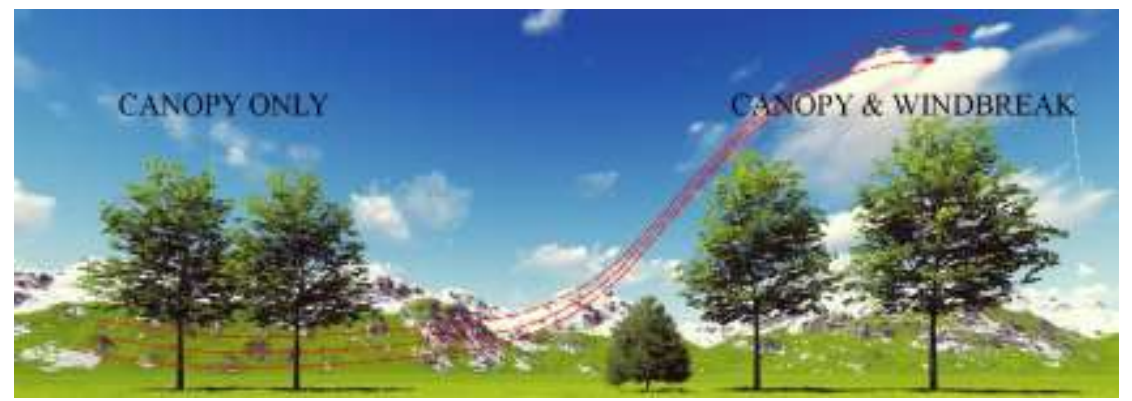

Fig. 6. The impact of air movement influenced by tree and shrubs design.

The best natural solar radiation control devices are trees, shrubs and canopies. Trees have a lot of leaf with large shadow, which bring many benefits for the environment in tropical countries. Getting its advantage helps better control solar radiation and terrestrial radiation [15]. With a layer of leaf canopy, it can normally absorb $80 \%$ of incoming visible radiation, whilst reflecting $10 \%$ and transmitting $10 \%$. Approximately $20 \%$ of infrared solar is absorbed with $50 \%$ being reflected and $30 \%$ is transmitted [16]. Consequently, to reduce solar radiation, these tree canopies are an integral (Fig. 6).

Leaves layers helps avoiding the energy reaching to the ground, thus reducing temperature and long wave radiation effectively. (Fig. 7) [17].

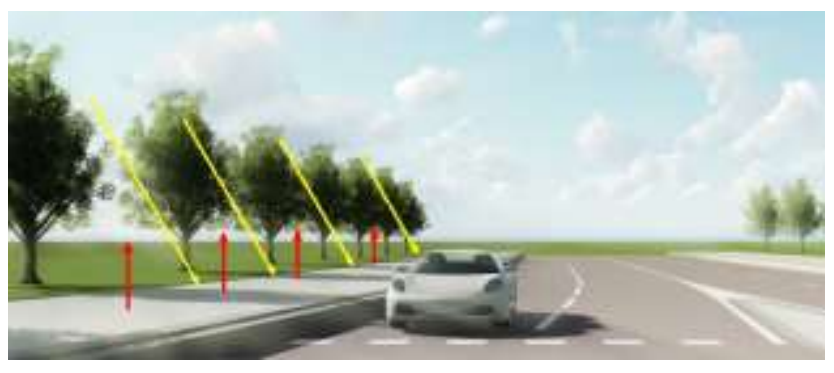

Fig. 7. Shades and tree layers help reducing the temperature of the ground's surface.

In order to deliver an optimal filtration, shading and temperature reduction underneath the canopy, these factors below should be considered in planting design:

Density from the canopy (leaf covers and branches).

- Height of the trees.

- $\quad$ Tree layer's characteristic in different seasons.

- $\quad$ Structure of the leaf layer and branches.

One of the microclimate elements that can be critical modified by planting layout is wind. It plays an important role in landscape and planting design for microclimate. Wind could carry heat away and be the most efficiency element affecting to the energy using. In addition, wind affects human in thermal comfort, temperature surrounding urban areas and also in energy used in buildings.

However, controlling wind is not an easy job ever. In general, it is possible to control wind by plants. There is a variation of controlling wind from choosing the different plant species to the arrangement of trees. [15]. But it should be considered that plants as natural elements which cannot be predicted absolutely in their growth, shape or effectiveness. Different combination of trees and shrubs can be used in a way that direct, reduce and deflect wind or increase the wind air flow with a substantial impact (Fig. 8). 


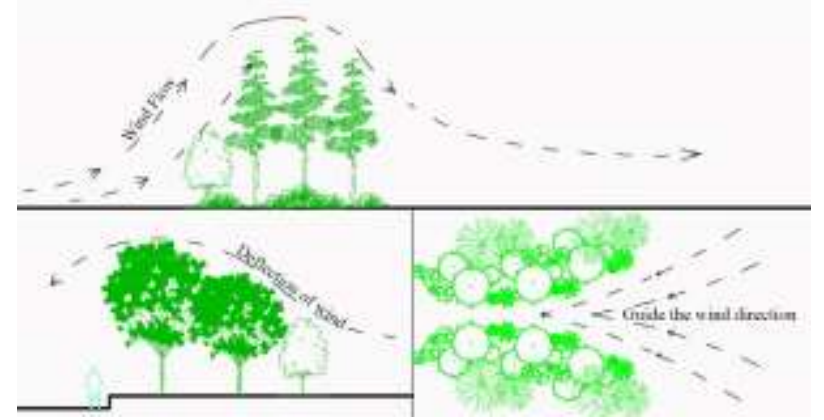

Fig. 8. Function of trees and shrub in moderating winds.

Wind velocity is reduced by tree generally. Reducing in wind velocity deliver a lowering of thermodynamic exchanges rate in the air layers allowing higher temperatures to prevail in the protected zone. There is a little or no difference between actual air temperatures in sun or shade where the condition is special with free air movement. Nevertheless, tree covered by shrubs reflecting air currents upward, there will be cooler temperatures in the shade [15]. Natural barriers broken by wind with low velocity such as trees and shrubs are often preferable to hard barriers (buildings, artificial blocks) because they do not create so much turbulence on the downstream side.

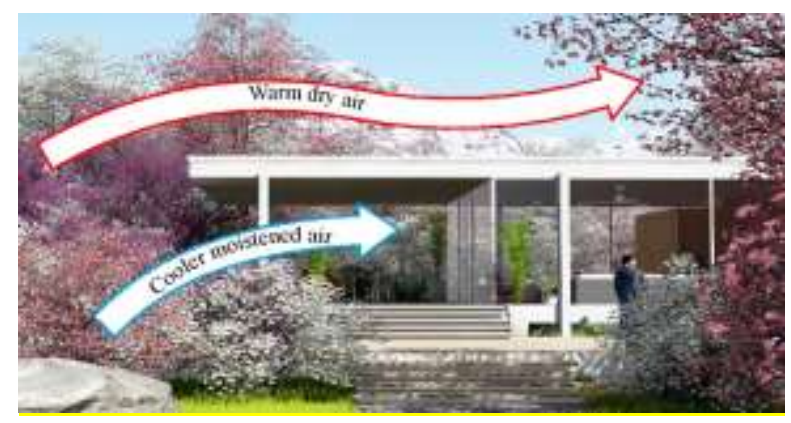

Fig. 9. Example: Effect of wind on evaporative cooling.

It can be observed that wind speed in the urban areas is reduced compared to surrounding rural areas. At pedestrian level, high wind speed is associated with streams in the corner or the windward vortex. If the area has many tall buildings, the effect will be increased which deflect faster moving upper air, thereby increasing wind speed at the pedestrian level. [18].

Applying on street branches, it is implied that different spacing between the buildings, comparing to building height may cause different flow patterns due to the interaction between upwind and downwind effects of buildings. Oke (1988) [19] used the height to width ratio $(\mathrm{H} / \mathrm{W})$ of street canyons to categorize wind flow into three flow regimes: (1) isolated roughness flow, (2) wake interference flow and (3) skimming flow, Figure 11.

In the first case, the buildings are relatively widely spaced $(\mathrm{HW}<0.3)$ and other buildings do not affect the flow around them, consider that if they were isolated obstacles (Fig. 10a). As distance increases downwind from a building, the sheltering effect decreases and eventually the jet merges with the flow and readjusts to its undisturbed form (Oke 1988) [19]. Therefore, in isolated roughness flow, the wind speed is expected to increase in the downwind direction behind a building created by the merging jet, before continuing on the next building downwind where it is kept to be stood still, increases resulting in a decrease in wind speed. 


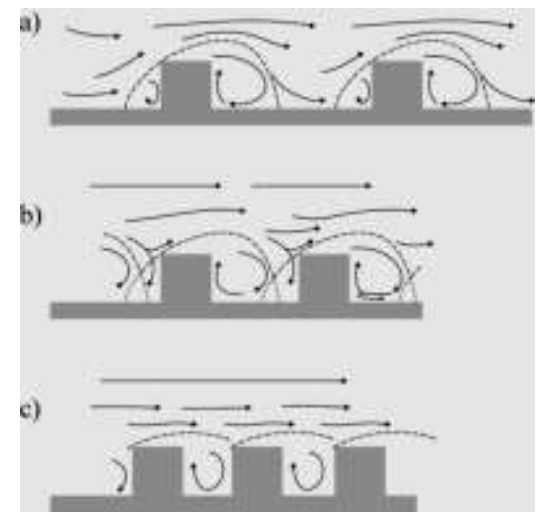

Fig. 10. Flow regimes indicated by Oke (1988): a) isolated roughness flow, b) wake interference flow, and c) skimming flow.

In the next case when spacing is medium $(0.3<\mathrm{HW}<0.7)$ the preceded zone of the upwind building interferes with the succeeded upwind stop zone of the next building downwind where it is called the wake interference flow (Fig. 10b). These arrangements become denser in the last case $(\mathrm{HW}<0.7)$, the main flow starts to slide above the roofs, driving a vortex in the cavity between the buildings beneath (Fig. 10c). In this situation, the bulk of the flow hardly pass inside the street canyon.

Furthermore, Plants also may be combined with landforms to moderate the airflow over the landscape or around the buildings (Fig.11). Landforms can direct wind and increase or decrease wind speed. However, it may be noticed that enhancing wind element by landform brings out a large effect. With other elements such as plants, landform probably give a hand in improving the vegetation.

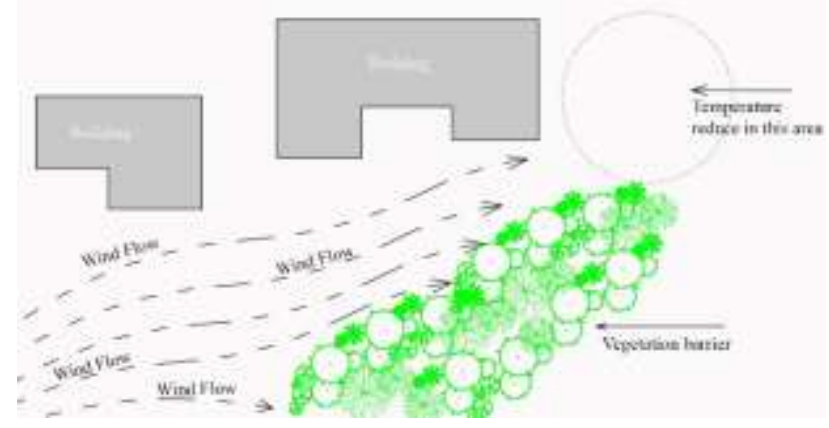

Fig. 11. Landscape and building acted as a barrier in directing the wind towards spot area to reduce temperature.

Another characterized factor affecting to planting design aspect are the size, orientation, porosity and proximity. Landscape elements used as wind barrier with lesser porous, impact greater on wind speed but in a small area of affected air. On the contrary, with more porous, the wind barrier having lower the effect on wind speed, but the area affected is larger. With the understanding, it is up to how we want it to be and how it can influence the micro surrounding based on the landscape materials chosen. In urban areas, creating a very small, very calm area, or a very large area with slightly lower wind speed deciding the quality and the level of human comfort.

Moreover, leaf transpiration process adds cool air into the temperature. Vapor converts into moisture through leaves, evaporative cooling effect occurs when heat is absorbed from the air. Humidity level is increased through the cooling effect. Thereby, in warm or dry 
climates, green canopy designing with multiple layers including shading creates cooling effect from leaf transpiration process. The cooler air can be withdrawn through wind flow and can expand to certain distance. These entities working together provide more comfortable air. It is necessary to increase the amount of plant cover to its fullest potential in landscape designing, which help maximizing the effect of evaporative cooling, instead of paved surfaces such as asphalt or concrete (Fig.11).

Featuring on layer canopy design to see microclimate modification effects are various and can be achieved basing on design parameters and scientific processes. The purpose of this study to create new microclimate environment covering the analysis, evaluation and understanding of site parameters, climate conditions, plant material characteristics, and environmental design approaches. Fig. 12 illustrates the basic guidelines on canopy design ideas to provide an effective high-quality environment in urban areas.

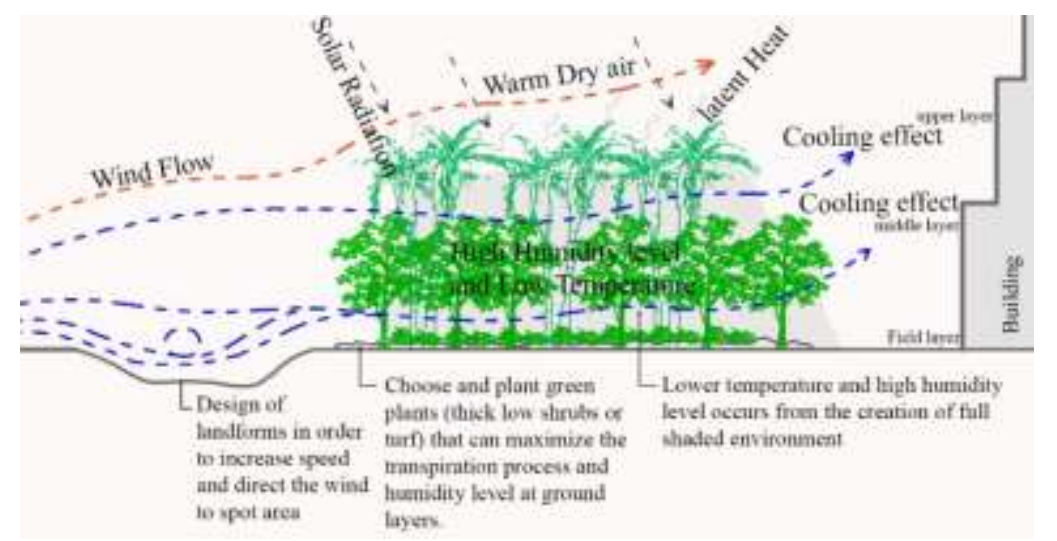

Fig. 12. Schematic diagram explaining the whole process of all four microclimate components modification based on planting design theories and guidelines.

\section{Conclusion}

At present, the importance of transforming and urban structure redesign was admitted in Vietnam as well as in the global world. In this stage, the optimal balance between economic, environmental and social aspects is crucial. In fact, this contribution helps reducing the harmful effects of heat island effects on humans and deliver new design rules for planning green cities. The utilization of multiple layers of trees at different heights creates a large shade area that increases high moisture levels, optimally reducing air temperatures in urban areas. Changes in density of leave layers will alter the solar radiation and reduce the heat transfer that optimizes the transpiration. Furthermore, different landforms have strong impact the wind speed blowing dry air above the canopy and providing humid air below the canopy to provide a favorable microclimate for the health of the residents. The concept of sustainable development has been more concerned by many scientists and researchers, studying microclimate elements, finding out new design rules and combine it with the tropical vegetation addressing a better strategy in UHI mitigation as well as create a new landscape green design.

\section{References}

1. L. Howard, The Climate of London: Deduced from Meteorological Observations Made in the Metropolis and at Various Places Around it (Harvey and Darton, London, 1833) 
2. General statistics of Vietnam, statistical documentation and service center (2016)

3. A.A. Van de Griend, M. Owen, International journal of remote sensing 14, 1119-1131 (1993)

4. G.S. Golany, Atmos. Environ. 30-3, 455-465 (1996)

5. C. Morris, I. Simmonds, N. Plummer, J. Appl. Meteorol. 40-2, 169-182 (2001)

6. R.A. Memon, D.Y. Leung, J. Environ. Sci. 22-12, 1902-1909 (2010)

7. Priyadarsini Rajagopalan, Wong Nyuk, D. Hien, K.W. Cheong, Sol. Energy 82-8, 727745 (2008)

8. E. Erell, D. Pearlmutter, T.T.J. Williamson, Urban microclimate: designing the spaces between buildings (Routledge, 2011)

9. S. Kato, H. Huang, J. Wind Eng. Ind. Aerodyn 97-7, 358-367 (2009)

10. J.A. Patz, D. Campbell-Lendrum, T. Holloway, J.A. Foley, Nature, 438-7066, 310-317 (2005)

11. T.R. Oke, Boundary Layer Climates, Psychology Press., Mausam 5-30, 134-138 (1992)

12. V. Ok, A. O"zsoy, V. Atl1, M. O“zgünler, N. Serteser, An analysis of the effect of design parameters on wind speed and flow type in an urban open space, TUBITAK (Turkish Scientific and Technical Research Council). INTAG-214 (1996)

13. N. Serteser, V. Ok, The effects of building parameters on wind velocity and air-flow type in the urban settlements. The seventh international conference on urban climate, Yokohama 29 (2009)

14. R.D. Brown, T.J. Gillespie, Microclimate Landscape Design: Creating Thermal Comfort and Energy Efficiency (John Wiley \& Sons New York, 1995)

15. G.O. Robinetee, Plants and their Enviromental Functions (Department of the Interior, Washington D.C., 1968)

16. M.F. Shahidan, K.M.S Mustafa, Tropical Microclimate Indicators for The Malaysian Urban Landscape. ASEAN Postgraduate Seminar in Built Environment (University of Malaya, Malaysia, 2005)

17. B. Kotzen, Journal of Arid Environments 55, 231-274 (2003)

18. B. Blocken, J. Carmeliet, Journal of Building Physics 28-2, 107-159 (2004)

19. Y. Nakamura, T.R. Oke, Atmospheric Environment 22-12, 2691-2700 (1988) 